\title{
Design of an Energy-Efficient Wireless Router Switch
}

\author{
Yuan Lulin \\ Wuhan University of Technology, Logistics \\ Engineering College \\ Hubei Wuhan, china \\ e-mail:1126966643@qq.com
}

\author{
Yang Jie \\ Wuhan University of Technology, Logistics \\ Engineering College \\ Hubei Wuhan,china \\ e-mail: 67445868@qq.com
}

\begin{abstract}
This paper introduces a kind of key technology of an energy-efficient wireless router switch design. The core of this technique is to monitor the flicker frequency of wireless network signal to realize the wireless router control. Our project team received data through experiments and analyzed it by mathematical methods to obtain the best detection scheme, where the detection period is three and a half minutes every time and the threshold is one hundred and twenty times.
\end{abstract}

Keywords-energy conservation; intelligent control; Wi-Fi I ntrusion Detection

\section{REACH BACKGROUND}

The wireless router would be open all the time whether it is connected to a network or not if nobody close it. According to statistics, In 2013 the global wireless network connection client devices (including the access point routers and gateways) shipments of more than 139000000, the router shipments of China nearly 50 million units In 2013. The waste caused by network idle energy is very large. With constant improvement of performance in router, scale, hardware integration, the power consumption of the router presents exponential growth $^{[1]}$, even in the idle state, network equipment takes a huge amount of energy. The problem of Internet energy-saving has gradually become a new research direction of the future network.

Some researches mainly rely on the energy saving in devices to realize the energy saving in router ${ }^{[2][3]}{ }^{[4]}$. Some studies use equipment dormancy to achieve energy-saving. Nedevschi and some others started from the resting condition and wake conditions, and put forward timer-driven strategy ${ }^{[5]}$. When the device's idle time exceeds a certain threshold, device will enter standby or hibernation, and when the device's sleeping time reaches a certain threshold, the equipment is awakened. Due to sleeping time is limited, energy saving condition is poor, and the data packet will be discarded during dormancy. WoA (wake-on-arrival) strategy is an improved strategy of timer-driven, and put specific interface equipment into working condition. When the interface detects a packet arriving, it will awake the device. But the equipments that can support WoA are few. Thoziyoor put forward a new strategy that use "dummy packet" to wake up device ${ }^{[6]}$. If A needs to wake up B, then A send a "dummy packet" to B firstly, and then wait for the $\delta$ time to communicate with B actually. Although this strategy reduces the packet losing rate, increasing the data packet delay.

In addition to energy saving in its own, some studies focus on the link between energy-saving equipment. Data shows when the Ethernet link rate increases from $100 \mathrm{Mbps}$ to $1 \mathrm{Gbps}$, power consumption has increased by $2 \sim 4 \mathrm{w}$, and when the Ethernet link rate increases to $10 \mathrm{Gbps}$, power consumption has increased by $10 \sim 20 \mathrm{w}{ }^{[7]}$. But the current Ethernet in power consumption in idle and non-idle condition is almost the same, measured data indicates that the link utilization of the Ethernet is mainly between $1 \%$ and $5 \%$. So when the link is in the low utilization rate, reducing the link rate can effectively reduce the energy consumption within the limited performance impact. Through automatically adjust the full-duplex transmission of Ethernet link exchange rate, adaptive link technology adapt to different link utilization, and make link transmission rate and the link load be proportional. At present, the research of the technology mainly focus on the affection of performance caused by rate of link switching and adaptive link rate strategy.

Some studies start from the improved routing protocol to achieve saving energy. Document proposed an algorithms that modify the OSPF protocol to achieve network energy saving. The algorithm enables the router to shut down part of a link in the case of low load. The algorithm is divided into 3 stages: the router is chosen as the output of its shortest path tree in the first stage, the second stage is the router's which is selected neighbor to execute a modified Dijkstra algorithm to detect whether the link can be closed, calculate the new path under Network Topology which has been modified. Data shows in the actual Internet, more than $60 \%$ of the links can be turned off.

This article will achieve the aim of energy saving from the external hardware and software.

\section{DATA COLLECTION EXPERIMENT}

\section{A. Purpose and Methods}

The purpose of the experiment is to get the best detection scheme, namely, use state of the network every time the detection and control of the power saving switch length threshold value, to ensure the accuracy and 
stability of the power saving switch of the wireless router control system.

The method of the experiment is to simulate different network environments, to record flash times of Wi-Fi indicator in the duration of all experiments using 51 single-chip.

\section{B. Experimental environment}

The experiment was carried out in the simulation of different networks in use. Based on people's net habits, test environment is divided into a total of 11 groups. To facilitate experiment, we recorded them as numbers.

Table 1 Groups of testing environment

\begin{tabular}{|c|c|}
\hline Circumstances 1 & No terminal device \\
\hline Circumstances 2 & Computer connected, none operation \\
\hline Circumstances3 & Computer connected and brows web \\
\hline Circumstances4 & $\begin{array}{l}\text { Computer connected and download } \\
\text { file }\end{array}$ \\
\hline Circumstances5 & $\begin{array}{c}\text { Mobile phone connected, none } \\
\text { operation }\end{array}$ \\
\hline Circumstances6 & $\begin{array}{l}\text { Mobile phone connected and brows } \\
\text { web }\end{array}$ \\
\hline Circumstances7 & $\begin{array}{l}\text { Mobile phone connected and } \\
\text { download file }\end{array}$ \\
\hline Circumstances8 & $\begin{array}{l}\text { Computer and mobile phone } \\
\text { connected, none operation }\end{array}$ \\
\hline Circumstances9 & $\begin{array}{l}\text { Computer and mobile phone } \\
\text { connected, only computer brows web }\end{array}$ \\
\hline Circumstances 10 & $\begin{array}{c}\text { Computer and mobile phone } \\
\text { connected, only mobile phone brows } \\
\text { web }\end{array}$ \\
\hline Circumstances11 & $\begin{array}{l}\text { Computer and mobile phone } \\
\text { connected, both brows web }\end{array}$ \\
\hline
\end{tabular}

\section{Experimental results and analysis}

\section{1) Experimental Data}

In the above-described 11 groups, respectively, we tested in the operating environment, obtaining the following experimental data.

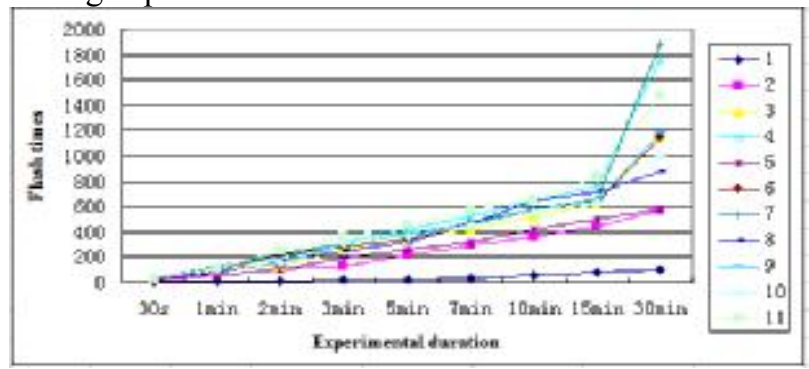

Figure 1 the number of wireless network indicator flashes line graph in different network states

\section{2) Data Analysis}

First, select network idle three test environments: 1, 2, and 5, then analyze their correlations. Experiment duration and the flashing numbers of Wi-Fi signal lights corresponding scatter plot is below.

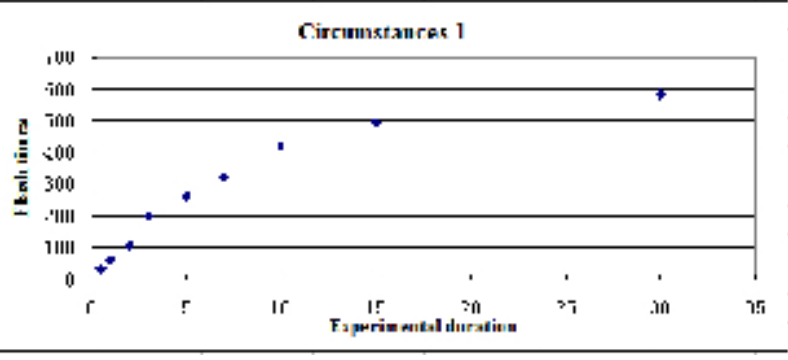

Figure 2 Flickering of wireless network light in environment 1

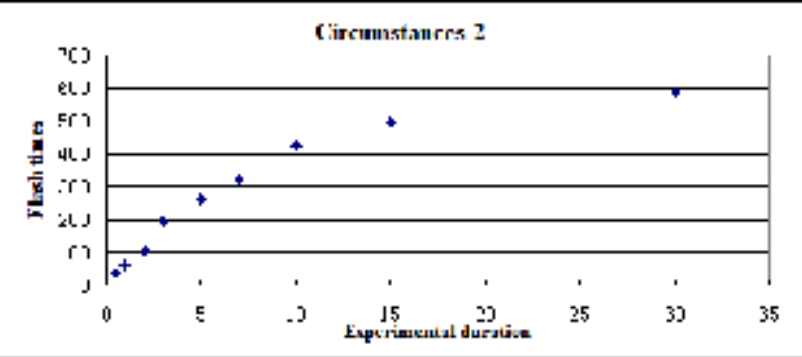

Figure 3 Flickering of wireless network light in environment 2

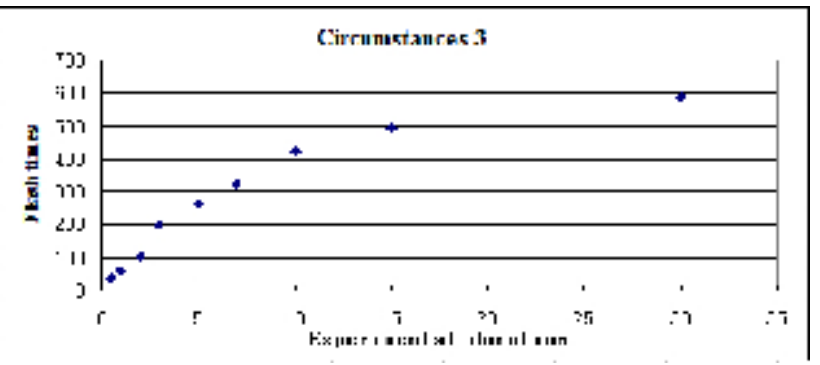

Figure 4 Flickering of wireless network light in environment 3

From the scatter plot, it is clear that there is a correlation between the flash numbers of wireless network signal lights and experimental time. We usually use the correlation coefficient ${ }^{\rho_{x y}}$ to express the degree of correlation between variables $\mathrm{x}$ and $\mathrm{y}$.

$$
\rho_{x y}=\frac{\sigma_{x y}}{\sigma_{x} \sigma_{y}}=\frac{E\left[\left(x-\mu_{x}\right)\left(y-\mu_{y}\right)\right]}{\sqrt{E\left[\left(x-\mu_{x}\right)^{2}\right] E\left[\left(y-\mu_{y}\right)^{2}\right]}}
$$

$$
\begin{aligned}
& \sigma_{x y}-\text { Covariance of random Variables } \mathrm{x}, \mathrm{y} ; \\
& \mu_{x}, \mu_{y} \_ \text {Mean of random Variables } \mathrm{x}, \mathrm{y} ;
\end{aligned}
$$




$$
\sigma_{x}, \sigma_{y} \_ \text {Standard deviation of random Variables }
$$
$\mathrm{x}, \mathrm{y}$.

From figure 8 line chart, we see that with increasing time of the test, the slope of the line showed a gradual increasing trend. The reason for being initially flat, then surge is that at the beginning the network is just in use, so the exchange is not frequent. After three minutes the data exchanges frequently, and the growth of Wi-Fi indicator flashes number surges.

The best detection scheme we finally get is: the each detected time of the network status is 3 minutes and 30 seconds, and the threshold is 120.If the flashing numbers of wireless networks lights during this period is below the threshold, the wireless router saving switch will automatically cut off power. The select of the best detection scheme successfully implements accurate and stable control of energy-saving switching power supply for wireless routers.

\section{HARDWARE AND SOFTWARE DESIGN OF ENERGY- SAVING SWITCH SYSTEM}

\section{A. Hardware Design of System}

AT89C2051RC chip is the control core of the system hardware. It also includes clock and reset circuit, power supply circuits, relays, infrared, etc. Composition is shown below.

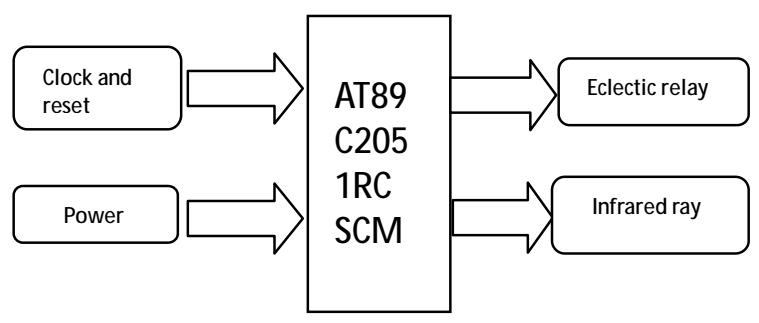

Figure 5 System hardware design block diagram

\section{B. Software Design of System}

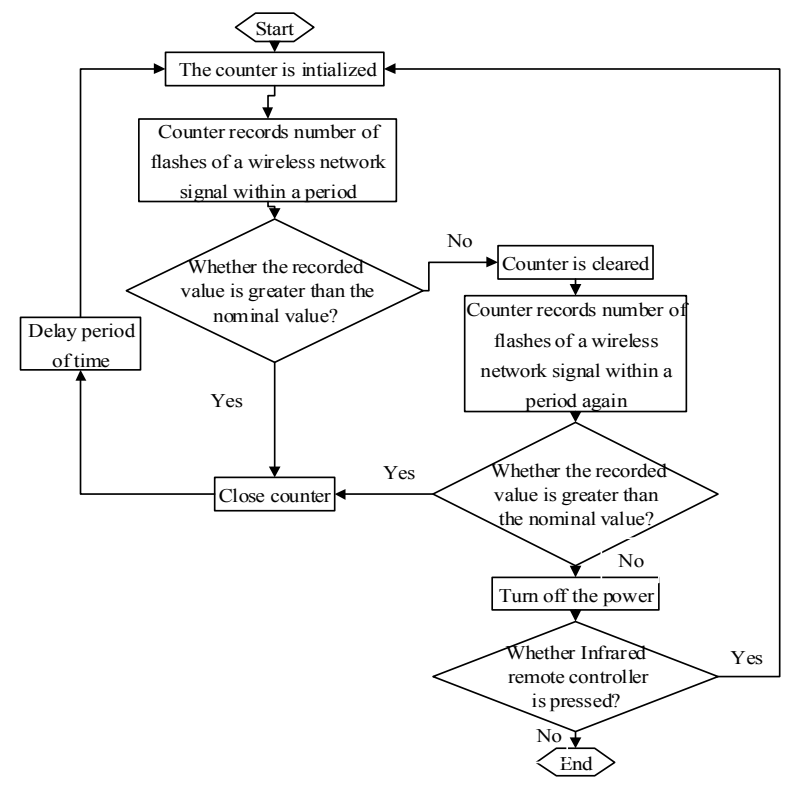

Figure 6 switch controlling flow chart

\section{Running Test of System}

\section{1) Power Analysis}

To determine the energy consumption of energy-saving switch wireless router, ordinary router and energy-saving switch router's power were tested. Wireless router model is Asus G32.We recorded the average power, and then averaged, obtaining the following experimental data.

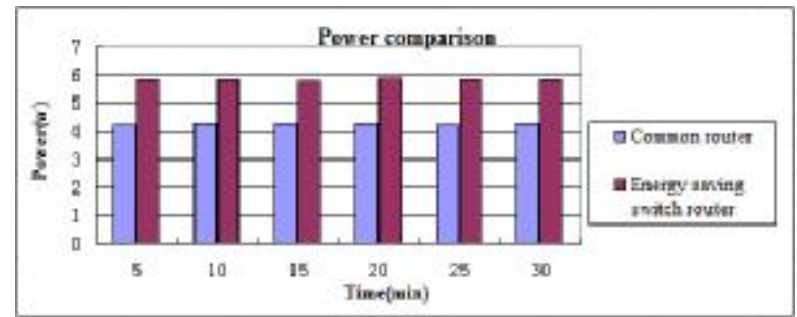

Figure 7 Power comparison diagram

Therefore, Ordinary router's average power is stably around $4.25 \mathrm{w}$, the average power of energy-saving switch router is stably around $5.85 \mathrm{~W}$, but in the same environment the running time of the former is more than 2 times to the latter. A wireless router will save power $13.359 \mathrm{kw} / \mathrm{h}$ one year which depends on the wireless router in experiment.

\section{2) Stability Analysis}

To test the stability of its operation. We applied for testing laboratory of automation institute in the Wuhan University of Technology. We tested it for half a month. In the process of testing, we simulated different networks use states, and the stability of the power-saving switch in the wireless router was tested. When the network is idle, no access to any device or devices to access a wireless router, but no network use, energy-saving switch wireless router will automatically turn off. That is, the wireless router energy-saving switch achieved a precise and stable control of the power supply. 


\section{3) Controllability analysis}

To test the controllability of the energy-efficient wireless router switch. We also applied for testing laboratory of automation institute in the Wuhan University of Technology. In the process of testing, When the wireless router's power automatically turn off, You can turn the power on again within a certain range through the infrared remote control, then the wireless router goes back to normal working condition.

\section{CONCLUSION}

In this paper, our project provides a highly efficient, precise, intelligent control scheme to solve the problem of energy waste of existing traditional wireless router, radiation pollution and other problems such as information security.

\section{ACKNOWLEDGEMENTS}

The research work was supported by Youth Funding Innovative Research Projects of Wuhan University of Technology(146818012)

\section{REFERENCES}

[1] Roberts L. Beyond moore's law: Internet growth trends [J]. IEEE Trans on Computer, 2000, 33(1): 177-119.

[2] Yao F, Demers A. A scheduling model for reduced CPU energy [C] //Proc of the 43rd Annual IEEE Symp on Foundations of Computer Science (FOCS'02). Piscataway, NJ: IEEE, 2002: 374-382.
[3] Augustine J, Irani S, Swamy C. Optimal power-down strategies [C] //Proc of the 45th Annual IEEE Symp on Foundations of Computer Science (FOCS'04). Piscataway, NJ: IEEE, 2004: 530-539.

[4] Pallipadi V, Starikovshiy A, The ondemand governor [C] //Proc of the Linux Symposium 2006, Piscataway, NJ: IEEE, 2006: 215-230.

[5] Nedevschi S, Popa L, Iannaccone G, et al, Reducing network energy consumption via sleeping and rare-adaptation [C] //Proc of the 5th USENIX Symp on Networked Systems Design and Implemenntation (NSDI'08). New York: ACM, 2008: 323-336.

[6] Thoziyoor S. Ahn J, Monchiero M, et al. A comprehensive memory modeling tool and its application to the design and analysis of future memory hieraechies [C] //Proc of ISCA'08. Piscataway, NJ: IEEE, 2008: 51-62.

[7] Gunaratne C, Christensen K, Nordman B. Managing energy consumption costs in desktop PCs and LAN switches with proxying split TCP connections and scaling of link speed [J]. Internetional Journal of Network Management, 2005, 15 (5): 297-310.

[8] Cianfrani A, Eramo V, Listanti M, et al. An energy saving routing algorithm for a green OSPF protocol [C] /Proc of the 29th IEEE Conf on Computer Communications Workshops (INFOCOM'10). Piscataway, NJ: IEEE, 2010: 1-5.

[9] Wang Yonghong; Li Xiuy. Wireless router control method and wireless router $[\mathrm{P}]$. China: CN102405674A, 2011-4-25.

[10] Guanl Lin. The method of adjusting the power, routers and network equipment [P]. China: CN101489294, 2009-7-22. 